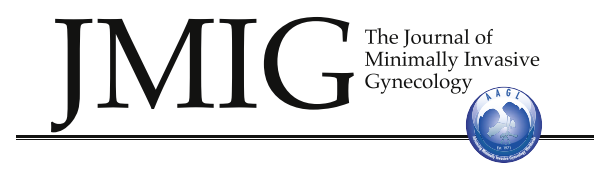

Case Report

\title{
Adenomyotic Cyst in a 25-Year-Old Woman: Case Report
}

\author{
Gaspare Cucinella, MD, PhD, Valentina Billone, MD*, Ilaria Pitruzzella, MD, \\ Attilio Ignazio Lo Monte, MD, PhD, Vincenzo Davide Palumbo, MD, and \\ Antonino Perino, MD, $\mathrm{PhD}$ \\ From the Department of Mother and Child (Drs. Cucinella, Billone, Pitruzzella, and Perino), and Department of Surgical and Oncological Sciences (Drs. Lo \\ Monte and Palumbo), University Hospital "P. Giaccone," University of Palermo School of Medicine, Palermo, Italy.
}

\begin{abstract}
Adenomyotic cysts are uncommon findings, usually in the context of diffuse adenomyosis and $<5 \mathrm{~mm}$ in diameter. Herein we report a 4.5 -cm adenomyotic cyst in a 25-year-old nulliparous woman with severe dysmenorrhea and pelvic pain. Transvaginal ultrasonography and magnetic resonance imaging revealed a well-circumscribed hypoechogenic mass in the posterior uterine wall, well separated from the uterine cavity. Pathologic analysis demonstrated that the cyst was lined with endometrial epithelium and stroma and was surrounded by smooth muscle hyperplasia. In the literature, we found 30 reports of cysts with similar characteristics. Because this cyst has not been clearly defined, it has been called by various names including adenomyotic cyst, cystic adenomyosis, and cystic adenomyoma. We believe this lesion should not be called an adenomyoma, but is more correctly called an adenomyotic cyst or, depending on age at onset, a juvenile adenomyotic cyst. Journal of Minimally Invasive Gynecology (2013) 20, 894-898 (C) 2013 AAGL. All rights reserved.
\end{abstract}

Keywords: $\quad$ Adenomyoma; Adenomyosis; Adenomyotic cyst; Cystic; Dysmenorrhea; Juvenile

DISCUSS You can discuss this article with its authors and with other AAGL members at http://www.AAGL.org/jmig-20-6-JMIG-D-13-00093R1

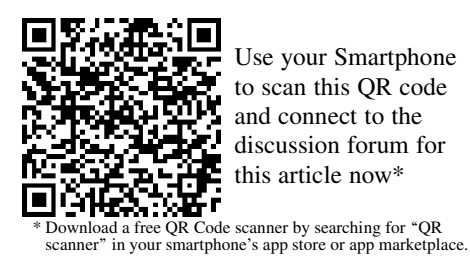

vention. Symptoms include severe and worsening secondary dysmenorrhea associated with menorrhagia and pelvic pain.

\section{Case Report}

A 25-year-old nulliparous woman came to our institution because of severe and worsening dysmenorrhea and abdominal cramping. Her first menses occurred when she was aged 12 years, and her menstrual cycle was regular (25 days). Dysmenorrhea began shortly after menarche; however, the pain was sufficiently relieved with use of analgesics (nimesulide or paracetamol).

In the past year, the pain had become more severe and refractory to any medical treatment. Typically, the pain began 1 week before menses and continued in the week afterward. The patient reported that the pain was more intense on the first day of each menstrual cycle. According to a visual analog scale ranging from 0 (no pain) to 10 (extreme pain), the severity of dysmenorrhea was 9 . The patient also had menorrhagia but no dyspareunia or dyschezia.

Pelvic examination revealed a normal vagina, vulva, and adnexae and an enlarged retroflexed uterus. Laboratory

Submitted February 5, 2013. Accepted for publication April 30, 2013.

Available at www.sciencedirect.com and www.jmig.org 


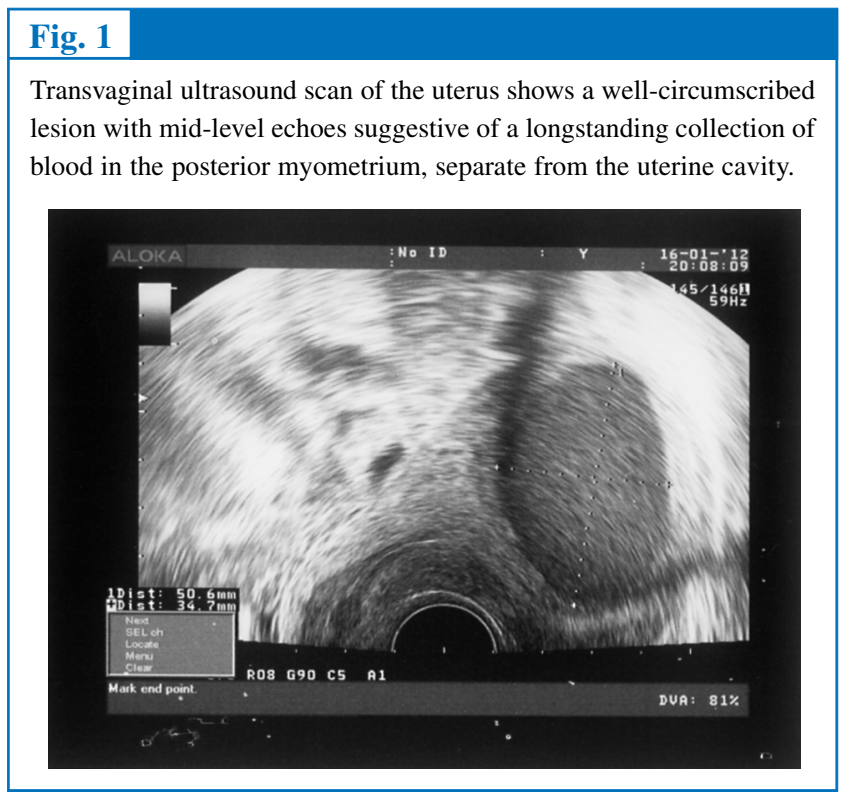

findings including complete blood cell count, complete metabolic panel, liver function tests, pancreatic enzyme levels, urinalysis, and erythrocyte sedimentation rate were within normal limits. CA 15.3, carcinoembryonic antigen, and $\beta$-human chorionic gonadotropin levels were normal (12.80 U/mL, $1.35 \mathrm{ng} / \mathrm{mL}$, and $1.20 \mathrm{mU} / \mathrm{mL}$, respectively), whereas CA 125 levels were slightly elevated (38.00 $\mathrm{U} / \mathrm{mL})$, and CA 19.9 level was high (88.19 U/mL). Transvaginal ultrasound, performed immediately before surgery, showed a well-circumscribed hypoechogenic mass, similar to an endometrioma, in the posterior uterine wall, well separated from the uterine cavity. The cyst cavity measured 4.5 $\times 2.4 \mathrm{~cm}$ in diameter and was surrounded by a thick capsule, for a total diameter of $5.0 \times 3.4 \mathrm{~cm}$ (Fig. 1). Transvaginal ultrasound performed during the luteal (secretory) phase of the menstrual cycle confirmed no evidence of communication between the lesion and the endometrium. Both ovaries appeared normal. Pelvic magnetic resonance imaging disclosed an retroflexed enlarged uterus $(7.2 \times 8.2 \times$ $8.7 \mathrm{~cm}$ ) and a rounded well-defined intramyometrial mass in the posterior wall of the uterine corpus. The mass measured $3.2 \times 4.5 \times 4.1 \mathrm{~cm}$, and was slightly hyperintense on T1- and T2-weighted images, with an internal fluidfluid level, i.e., layering of simple and hemorrhagic or proteinaceous fluids (Fig. 2). Small masses in both ovaries (right, $12 \mathrm{~mm}$; left, $7 \mathrm{~mm}$ ) with the same findings as those of the intramyometrial mass were noted.

Operative laparoscopy was performed after 3 months, on day 24 of the menstrual cycle, with the patient under general anesthesia. With the patient in a lithotomy position $( \pm 20-$ degree Trendelenburg position), pneumoperitoneum was induced using a Veress needle, and trocars were inserted. At laparoscopic observation, the posterior wall of the uterine corpus demonstrated an altered profile. After the external

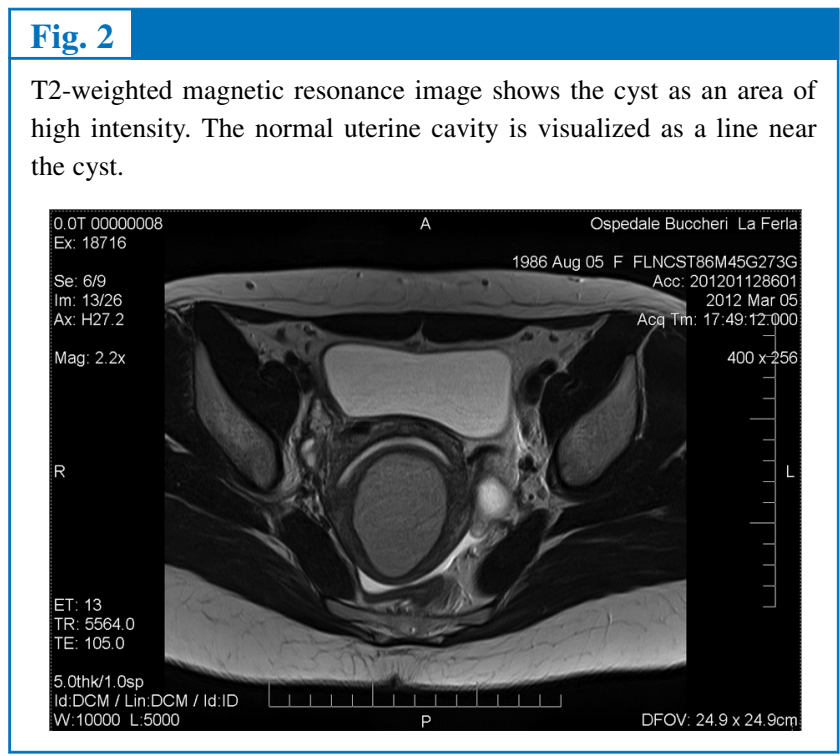

layers of the mass were incised, a chocolate-colored fluid was drained. During the surgical procedure, intrauterine methylene blue injections demonstrated no communication between the endometrial cavity and the cyst, and both fallopian tubes were patent.

The surgical procedure consisted of radical excision of the adenomyosis, leaving a $1-\mathrm{cm}$ margin of tissue above the endometrium and a 1-cm margin of tissue below the serosal surface, with subsequent reconstruction of the uterus. The enlarged uterus was bisected using a monopolar hook, from the serosal surface of the fundus, in the midline and in the sagittal plane, down through the adenomyosis. Thus, the entire extent of the adenomyosis was clearly visible, with the crucial landmarks of the endometrium and the serosal surface always in clear view. The adenomyotic tissues were grasped and excised from the surrounding myometrium, leaving $1 \mathrm{~cm}$ of myometrial tissue from the serosa above and the endometrium below. Thereafter, the normal myometrium was closed using interrupted absorbable suture (Fig. 4). Small foci of endometriosis were detected in the right ovary and the pouch of Douglas, and were treated using bipolar electrocautery. Operative time was 70 minutes, and blood loss was $<20 \mathrm{~mL}$. The postoperative course was normal, and the patient was discharged 2 days after the intervention.

Postoperatively, a gonadotropin-releasing hormone agonist was administered for 3 months. At menstruation, the patient had no dysmenorrhea.

At macroscopic examination, the lesion measured $4.5 \mathrm{~cm}$ in diameter. Biopsy specimens demonstrated endometrial epithelium and stroma lining the inner cyst wall, bordered by a region of myometrial hyperplasia. Hemorrhage and hemosiderin-laden macrophages were seen in association with endometrial sloughing (Fig. 3). 


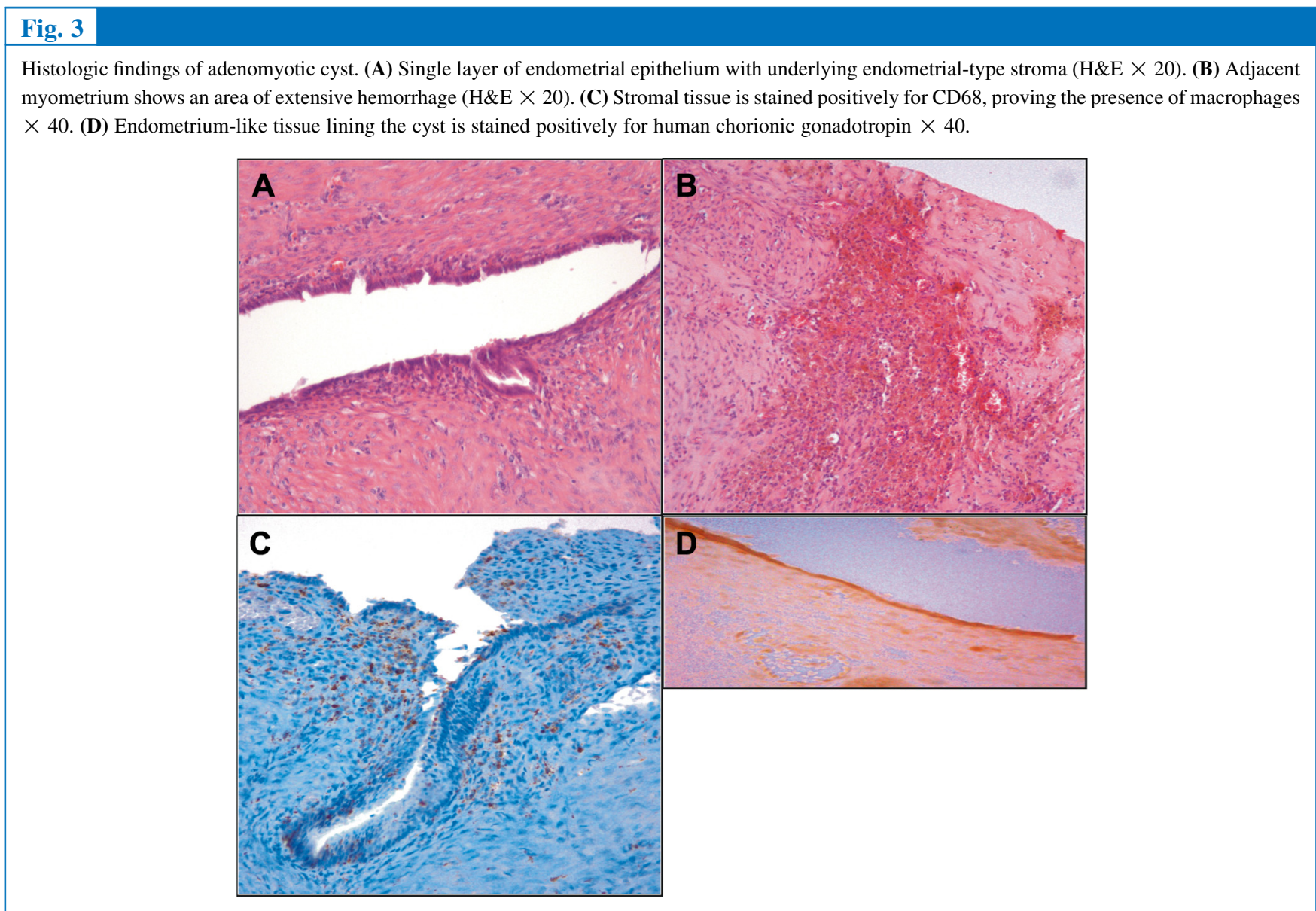

\section{Discussion}

Cystic structures within the uterine cavity are uncommon [2,3]. Uterine cysts are classified into 2 main groups: congenital and acquired. Acquired cysts include cystic degeneration of uterine leiomyoma, cystic adenomyosis, and serosal cysts. Congenital cysts derive from müllerian (paramesonephric) duct anomalies such as a noncommunicating rudimentary horn and a unicornuate uterus or can be represented by wolffiann duct (mesonephric) and müllerian duct cysts [4].

Large adenomyotic cysts ( $\geq 1 \mathrm{~cm}$ in diameter) are rare. Although any site in the uterus may be involved, the posterior wall is most often affected [5].

There is no agreement about the definition of adenomyotic lesions. Several terms have been used to define these large cysts including adenomyotic cyst [6-8], cystic adenomyoma [9-11], and cystic adenomyosis [12,13]. According to Takeuchi et al [14], adenomyoma is a neoplastic tumor classified as a benign mixed epithelial-nonepithelial tumor. The designation of adenomyoma should be reserved for polypoid lesions in which the stromal component is almost exclusively composed of smooth muscle. The term adenomyoma is not recommended to define a solitary myometrial nodule of adenomyosis [15].
Furthermore, there is doubt about the nature of such rare voluminous cysts in young women. Considering patient age at symptom occurrence, some authors have classified these lesions into 2 categories: the adult and the juvenile forms. The adult form seems to result from trauma at the endometrium-myometrium interface, e.g., after uterine instrumentation [16]. The juvenile form is considered a congenital disease that develops from duplication and persistence of ductal müllerian tissue in a critical area, close to the root of the round ligament, possibly related to gubernaculum dysfunction [17]. Other authors [3,14] have described both the juvenile and adult forms as of acquired origin, probably related to a cystic variant of adenomyosis.

At histologic analysis, adenomyotic cysts are cavities lined by endometrial epithelium [18]. The stroma below the epithelium is thin throughout the cyst and contains red blood cells and hemosiderin-laden macrophages. The stromal cells are morphologically similar to those of the endometrium, as in endometriosis.

In conclusion, taking into consideration the relevant literature, the clinical findings, and the pathologic features, we consider the large cyst in our patient to be an acquired adenomyotic cyst. That the cyst was acquired is suggested by onset of dysmenorrhea long after menarche, absence of other 
Fig. 4

(A) Laparoscopic view of adenomyotic cyst located in the posterior uterine wall. (B and C) After incision, the chocolate-colored fluid was drained. (D) Adenomyotic cyst was grasped and excised from surrounding myometrium leaving a myometrial thickness, from the serosa above and the endometrium below, of $1 \mathrm{~cm}$. (E) The cystic wall. (F) Uterine myometrium was closed using an interrupted absorbable suture.
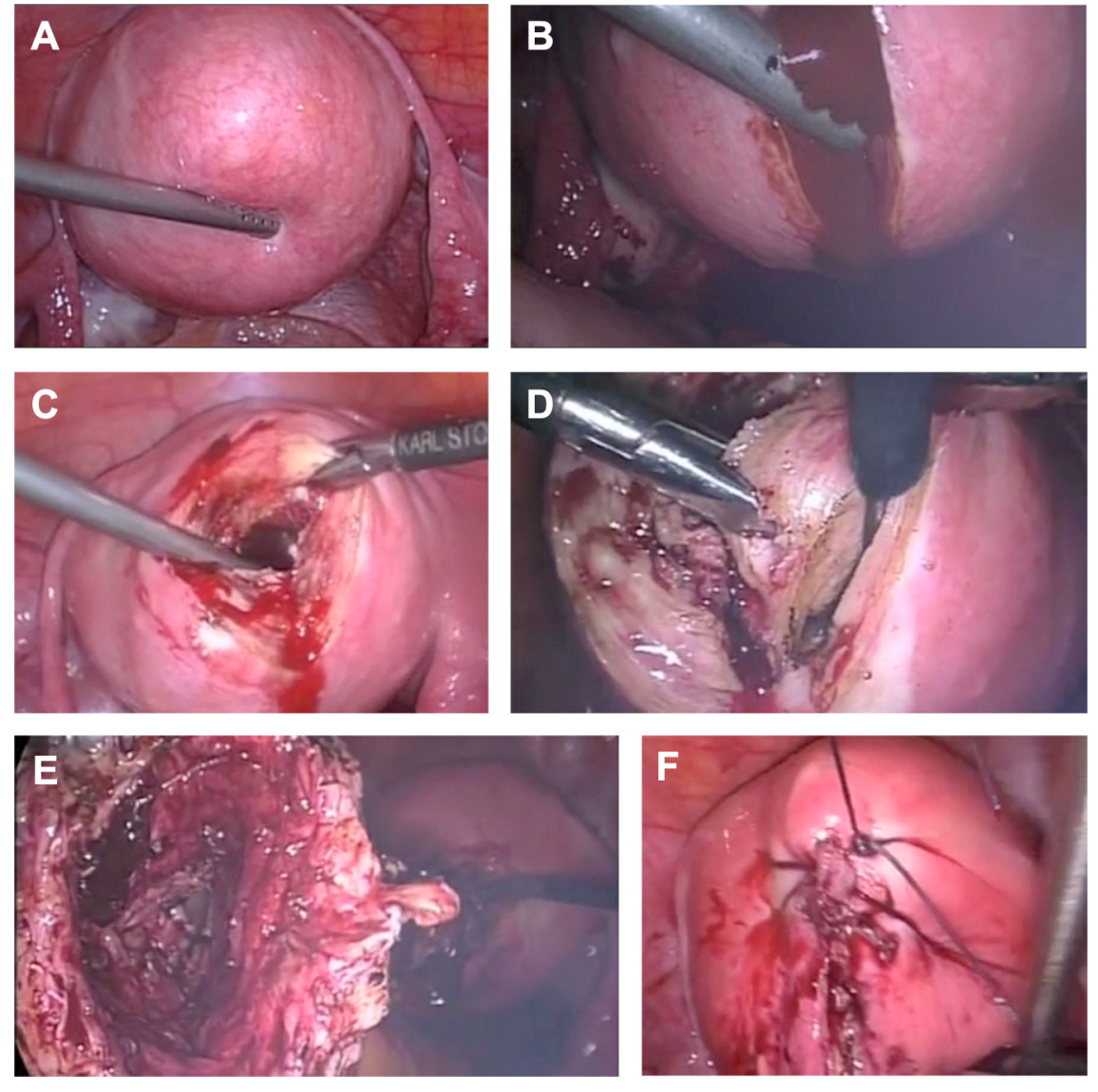

associated congenital defects, absence of any communication with the uterine cavity, and location far from the round ligament.

The presence of endometrium surrounding the internal cavity, composed of a single columnar layer accompanied by endometrial stroma, hemorrhage, and hemosiderinladen macrophages, is a clear characteristic of adenomyosis. We believe this lesion should not be called an adenomyoma because it did not have a relevant smooth muscle component; rather, it is more correctly called an adenomyotic cyst. Because of the age at onset, it could more exactly be called a juvenile adenomyotic cyst.

Further case reports are required to better understand the pathogenesis and clinical characteristics of this rare disorder.

\section{References}

1. Slezak P, Tillinger KG. The incidence and clinical importance of hysterographic evidence of cavities in the uterine wall. Radiology. 1976; 118:581-586.
2. Muzii L, Marana R, Angioli R, et al. Histologic analysis of specimens from laparoscopic endometrioma excision performed by different surgeons: does the surgeon matter? Fertil Steril. 2011;95:2116-2119.

3. Dogan E, Gode F, Saatli B, Secil M. Juvenile cystic adenomyosis mimicking uterine malformation: a case report. Arch Gynecol Obstet. 2008; 278:593-595.

4. Kim NR, Cho HY, Ha SY. Intramyometrial uterine cysts with special reference to ultrastructural findings: report of two cases. J Obstet Gynaecol Res. 2011;37:259-263.

5. Levgur M. Diagnosis of adenomyosis. J Reprod Med. 2007;52:177-193.

6. Ejeckam GC, Zeinab OA, Bobeck HE, Salman M, Bobeck HE. Giant adenomyotic cyst of the uterus. Br J Obstet Gynaecol. 1993;100: 596-598.

7. Tamura M, Fukaya T, Takaya R, Ip CW, Yajima A. Juvenile adenomyotic cyst of the corpus uteri with dysmenorrhea. Tohoku J Exp Med. 1996;178:339-344.

8. Kamio M, Taguchi S, Oki T, et al. Isolated adenomyotic cyst associated severe dysmenorrhea. J Obstet Gynecol Res. 2007;33:388-391.

9. Buerger PT, Petzing HE. Congenital cysts of the corpus uteri. Am J Obstet Gynecol. 1954;67:143-151.

10. Nabeshima H, Murakami T, Terada Y, et al. Total laparoscopic surgery of cystic adenomyoma under hydroultrasonographic monitoring. J Am Assoc Gynecol Laparosc. 2003;10:195-199.

11. Wang JH, Wu RJ, Xu KH, Lin J. Single large cystic adenomyoma of the uterus after cornual pregnancy and curettage. Fertil Steril. 2007;88: 965-967. 
12. Troiano RN, Flynn SD, McCarthy S. Cystic adenomyosis of the uterus: MRI. J Magn Reson Imaging. 1998;8:1198-1202.

13. Iribarne C, Plaza J, De la Fuente P, Garrido C, Garzon A, Olaizola JI. Intramyometrial cystic adenomyosis. J Clin Ultrasound. 1994;22: 348-350.

14. Takeuchi H, Kitade M, Kikuchi I, Kumakiri J, Kuroda K, Jinushi M. Diagnosis, laparoscopic management and histopathological findings of juvenile cystic adenomyoma: a review of nine cases. Fertil Steril. 2010; 94:862-868.

15. Silverberg SG, Kurman RJ. Tumor-like lesions. In: Silverberg SG, Kurman RJ, editors. Atlas of Tumor Pathology: Tumors of the Uterine Corpus and Gestational Trophoblastic Disease. Third series, fascicle
3. Washington, DC: Armed Forces Institute of Pathology; 1992. p. 191-218.

16. Kriplani A, Mahey R, Agarwal N, Bhatla N, Yadav R, Singh MK. Laparoscopic management of juvenile cystic adenomyoma: four cases. $J$ Minim Invasive Gynecol. 2011;18:343-348.

17. Acien P, Acien M, Fernandez F, Jose Mayol M, Aranda I. The cavitated accessory uterine mass: a mullerian anomaly in women with an otherwise normal uterus. Obstet Gynecol. 2010;116:1101-1109.

18. Takeda A, Sakai K, Mitsui T, Nakamura H. Laparoscopic management of juvenile cystic adenomyoma of the uterus: report of two cases and review of the literature. J Minim Invasive Gynecol. 2007;14: 370-374. 